\title{
Optimal order a posteriori error estimates for a class of Runge-Kutta and Galerkin methods
}

\author{
Georgios Akrivis ${ }^{1}$, Charalambos Makridakis ${ }^{2}$, \\ Ricardo H. Nochetto ${ }^{3}$ \\ 1 Computer Science Department, University of Ioannina, 45110 Ioannina, Greece; \\ e-mail: akrivis@cs.uoi.gr \\ 2 Department of Applied Mathematics, University of Crete, 71409 Heraklion- \\ Crete, Greece and Institute of Applied and Computational Mathematics, FOR- \\ TH, 71110 Heraklion-Crete, Greece; e-mail: makr@tem.uoc.gr \\ 3 Department of Mathematics and Institute for Physical Science and Technology, \\ University of Maryland, College Park, MD 20742, USA; \\ e-mail: rhn@math.umd.edu
}

Received: January 1, 2008 / Revised version: July 3, 2009

Summary We derive a posteriori error estimates, which exhibit optimal global order, for a class of time stepping methods of any order that include Runge-Kutta Collocation (RK-C) methods and the continuous Galerkin (cG) method for linear and nonlinear stiff ODEs and parabolic PDEs. The key ingredients in deriving these bounds are appropriate one-degree higher continuous reconstructions of the approximate solutions and pointwise error representations. The reconstructions are based on rather general orthogonality properties and lead to upper and lower bounds for the error regardless of the time-step; they do not hinge on asymptotics. ${ }^{1}$

Mathematics Subject Classification (1991): 65M15, 65M50

\footnotetext{
Correspondence to: Ch. Makridakis

1 The first author was partially supported by an 'EPEAEK II' grant funded by the European Commission and the Greek Ministry of National Education.

The second author was partially supported by the RTN-network HYKE, HPRNCT-2002-00282, the EU Marie Curie Dev. Host Site, HPMD-CT-2001-00121 and the program Pythagoras of EPEAEK II.

The third author was partially supported by NSF grants DMS-0505454 and DMS0807811 .
} 
Key words Runge-Kutta methods, collocation methods, continuous Galerkin method, time reconstruction, a posteriori error analysis, parabolic equations.

\section{Introduction}

We consider Runge-Kutta collocation type time-stepping schemes of any order $q \geq 1$, along with associated Galerkin methods, for parabolic partial differential equations (PDEs) and stiff ordinary differential equations (ODEs) of the form

$$
\left\{\begin{array}{l}
u^{\prime}(t)+A u(t)=B(t, u(t)), \quad 0<t<T, \\
u(0)=u^{0}
\end{array}\right.
$$

Hereafter $A$ is a positive definite, selfadjoint, linear operator on a Hilbert space $(H,\langle\cdot, \cdot\rangle,|\cdot|)$ with domain $D(A)$ dense in $H$, that dominates a (possibly) nonlinear operator $B(t, \cdot): D(A) \rightarrow H, t \in[0, T]$, and $u^{0} \in H, V:=D\left(A^{1 / 2}\right)$. We extensively study the linear case corresponding to $B(t, u)=f(t)$ with a given $f:[0, T] \rightarrow H$. We present a general framework for a posteriori error analysis based on the novel idea of time reconstruction of the approximate solution and of appropriate error representation equations that are derived with its aid. The resulting error estimates, valid for any $q \geq 1$, can be obtained by employing PDE stability techniques.

Error control for ODEs and evolution PDEs is a fundamental topic in scientific and engineering computing. The former has been developed since the 60's whereas the latter is much more recent. RungeKutta-Fehlberg methods are now standard high order methods for ODEs that estimate local truncation errors. For PDEs, instead, most of the available results are limited to low order time-stepping methods and to discontinuous Galerkin-type time discrete schemes. A primary tool to develop a posteriori error estimates for PDEs has been duality, either by estimating stability factors analytically $[9,10,27]$, or computationally upon solving a backward linear problem $[5,11,13$, 14,17]. The latter is mostly heuristic, even for linear equations of the form (1.1), and difficult to implement efficiently for large problems in several space dimensions. It provides however a general procedure to deal with possible error accumulation and long time behavior. Recently, we have developed a completely rigorous alternative to duality, mainly for general dissipative problems of the form (1.1). Optimal order error estimates have been derived for (1.1) by means of the energy method and the variation of constants (Duhamel) formula for both 
dG [23] and Crank-Nicolson schemes [2]. These are higher order extensions of the optimal a posteriori error analysis by Nochetto, Savaré and Verdi for the backward Euler method for a class of nonlinear gradient flows much more general than (1.1) and for which duality does not apply in general [24].

A posteriori error analysis for higher order Runge-Kutta methods seems to be lacking. We are only aware of rather interesting heuristic techniques based on asymptotic expansions and estimation of local truncation errors in the context of ODEs, see, e.g., $[7,16$, $25,26]$ and their references. In this paper we fill in this gap upon developing a posteriori error estimates for Runge-Kutta Collocation methods (RK-C), the most important class of implicit RK schemes (IRK), as well as related continuous Galerkin methods (cG). The analysis is in the spirit of, and indeed extends, our previous work [2] for Crank-Nicolson methods. The main contributions of this paper are as follows:

- We present a unified approach based on the orthogonality property

$$
\int_{0}^{1} \prod_{i=1}^{q}\left(\tau-\tau_{i}\right) d \tau=0
$$

for the collocation nodes $\left\{\tau_{i}\right\}_{i=1}^{q}$, which applies to $\mathrm{cG}$ (see $\S 3$ ) and RK-C (see $\S 4$ ).

- We introduce the time reconstruction $\widehat{U}$ of the discrete solution $U$, which is one-degree higher than $U$, is globally continuous but constructed locally (in one or two consecutive intervals), and extracts information about the local error without resorting to asymptotics (and thus to small time-steps); see $\S 2.1, \S 3.1$, and $\S 4.4$.

- We derive upper and lower a posteriori error estimates, which exhibit no gaps and possess explicit stability constants for the linear case; see $\S 2.2$. We apply the energy method, but any technique for error analysis such as the duality method could be used instead once $\widehat{U}$ has been constructed.

We emphasize that the main purpose of this paper is to introduce a new methodology for performing a posteriori error analysis for RungeKutta schemes of any order $q$. We insist on linear equations, for which our results are optimal, but not on the derivation of sharp estimates for nonlinear problems, a very delicate task that is heavily problem dependent. Similarly, we do not insist on conditional estimates in the present work; see Remark 3.4 regarding our assumptions.

Our unified approach hinges on suitable projection operators $\Pi_{q-1}$ and $\widehat{\Pi}_{q}$ onto spaces of piecewise polynomials of degree $q-1$ and $q$, 
respectively, determined by the collocation nodes $\left\{\tau_{i}\right\}_{i=1}^{q}$ in (2.7). In this vein, both RK-C and cG can be written in the following form provided $B(t, u)=f(t)$

$$
U^{\prime}(t)+\Pi_{q-1} A U(t)=\Pi_{q-1} f(t) .
$$

This is our abstract point of departure in $\S 2$, where we define the time reconstruction $\widehat{U}$ of $U$ with the help of $\widehat{\Pi}_{q}$. We observe now, but elaborate further in $\S 2$, that a naive use of the linear error-residual equation

$$
e^{\prime}(t)+A e(t)=-R(t)
$$

for the error $e=u-U$ and residual $R$ of the approximate solution $U$, would be suboptimal. This is because $R(t)=O\left(k^{q}\right)$ while the expected order for $e$ is $O\left(k^{q+1}\right)$, where $k$ denotes the time step. It is thus desirable to have an error equation with optimal order residual. To achieve this crucial goal, we choose to compare $u$ with the reconstruction $\widehat{U}$ of $U$ rather than with $U$ itself. The proper choice of $\widehat{U}$ is highly nontrivial and is the main contribution of this paper. In fact we require that $\widehat{U}$ satisfies the following crucial but competing properties:

- $\widehat{U}$ should be easily computable from $U$, and the operators and data in (1.1), within one or two consecutive time intervals and so locally for any time steps;

- $\widehat{U}$ should be globally continuous and one-degree higher than $U$;

- $\widehat{U}$ should extract relevant information from $U$ that dictates the local error;

- The residual $\widehat{R}$ associated with $\widehat{U}$ should be easy to evaluate in terms of $\widehat{U}-U$.

The concept of reconstruction might appear, at first sight, to be related to the technique of Zadunaisky [28] for error control of ODEs. The idea in [28] is to consider a perturbed ODE satisfied by a polynomial constructed by interpolating the approximate values on several time intervals in order to derive a heuristic estimate of the error. On the other hand, Runge-Kutta-Fehlberg methods also increase the order by one and find a computational estimate of the local truncation error. In both cases, the ensuing estimates are based on asymptotics and thus can only be rendered rigorous for small time steps. We stress that our reconstruction $\widehat{U}$ is not a higher order approximation of $u$ than $U$, which is another important difference with these two rather popular techniques. We also mention the related technique of elliptic reconstruction, introduced for a posteriori error analysis of space discrete finite element approximations in [22]. 
It turns out that the error $\hat{e}=u-\widehat{U}$ satisfies the equation

$$
\hat{e}^{\prime}(t)+A \hat{e}(t)=-\widehat{R}(t)
$$

involving the residual $\widehat{R}$, which is dominated by the optimal a posteriori quantity $U-\widehat{U}$. We stress that once such an equation for $\hat{e}$ is at our disposal, any stability technique available for the PDE under study can be used to derive estimates of the error. We derive, for simplicity, energy based upper and lower error estimates, with emphasis on the norms $L^{\infty}([0, T] ; H)$ and $L^{2}([0, T] ; V)$ rather than nodal values. We report these results for linear equations in Theorem 2.1 and for nonlinear equations in Theorems 3.1 and 4.1. We also give explicit expressions for $U-\widehat{U}$ in Corollary 2.1. Under restrictive compatibility conditions it is known that the order of convergence at the nodes (superorder) might be higher. For a posteriori error estimates related to superorder we refer to the forthcoming work [3]; see also section 4.2 below.

The paper is organized as follows. In $\S 2$ we present an abstract framework for time discretization and time reconstruction, with emphasis on the simpler linear case. In $\S 3$ we apply these results to cG and extend them to nonlinear equations. In $\S 4$ we deal with RK-C, and discuss the relation between classical order and stage order. In fact, viewing RK-C methods as collocation or Galerkin-type methods clarifies the connection between stage order and order of convergence in $L^{\infty}([0, T] ; H)$. The latter is $\mathcal{O}\left(k^{q+1}\right)$ because the approximate solution is a piecewise polynomial of degree $q$; note that a similar a priori bound for the error at the intermediate stages is obtained in $[20,21]$. Even though the emphasis is on RK-C methods, we discuss cG first because it is simpler to describe and study than RK-C.

\section{Time-Stepping Schemes and Time Reconstruction}

Let $0=t^{0}<t^{1}<\cdots<t^{N}=T$ be a partition of $[0, T], J_{n}:=$ $\left(t^{n-1}, t^{n}\right]$, and $k_{n}:=t^{n}-t^{n-1}$. Now, let $\mathcal{V}_{q}, q \in \mathbb{N}$, be the space of continuous functions that are piecewise polynomials of degree $q$ in time, i.e., $\mathcal{V}_{q}$ consists of continuous functions $g:[0, T] \rightarrow D(A)$ of the form

$$
\left.g\right|_{J_{n}}(t)=\sum_{j=0}^{q} t^{j} w_{j}, \quad w_{j} \in D(A) .
$$

We denote by $\mathcal{V}_{q}\left(J_{n}\right)$ the space of restrictions to $J_{n}$ of elements of $\mathcal{V}_{q}$. The spaces $\mathcal{H}_{q}$ and $\mathcal{H}_{q}\left(J_{n}\right)$ are defined analogously by requiring $w_{j} \in$ $H$. In the sequel we are mainly interested in the continuous Galerkin 
(cG) and Runge-Kutta collocation (RK-C) time-stepping schemes. We cast these methods in a wider class of schemes formulated in a unified form with the aid of a projection operator

$$
\Pi_{\ell}: C^{0}([0, T] ; H) \rightarrow \oplus_{n=1}^{N} \mathcal{H}_{\ell}\left(J_{n}\right),
$$

which does not enforce continuity at $\left\{t^{n}\right\}_{n=1}^{N}$. The time discrete approximation $U$ to the solution $u$ of (1.1) is then defined as follows: We seek $U \in \mathcal{V}_{q}$ satisfying the initial condition $U(0)=u^{0}$ as well as

$$
U^{\prime}(t)+\Pi_{q-1} A U(t)=\Pi_{q-1} f(t) \quad \forall t \in J_{n}
$$

for $n=1, \ldots, N$. Since all terms in this equation belong to $\mathcal{H}_{q-1}\left(J_{n}\right)$, (2.2) admits the Galerkin formulation

$$
\begin{array}{r}
\int_{J_{n}}\left[\left\langle U^{\prime}, v\right\rangle+\left\langle\Pi_{q-1} A U, v\right\rangle\right] d t=\int_{J_{n}}\left\langle\Pi_{q-1} f, v\right\rangle d t \\
\forall v \in \mathcal{H}_{q-1}\left(J_{n}\right),
\end{array}
$$

for $n=1, \ldots, N$. We use mainly (2.2), but (2.3) is also of interest because it provides a connection of this class of methods to the Galerkin schemes. In fact, we show later that the continuous Galerkin method corresponds to the choice $\Pi_{q-1}:=P_{q-1}$, with $P_{\ell}$ denoting the (local) $L^{2}$ orthogonal projection operator onto $\mathcal{H}_{\ell}\left(J_{n}\right)$ for each $n$; in this case $\Pi_{q-1}$ in (2.3) can be replaced by the identity. The Runge-Kutta collocation methods constitute the most important class of time-stepping schemes described by this formulation. We will see later that all RK-C methods with pairwise distinct nodes in $[0,1]$ can be obtained by choosing $\Pi_{q-1}:=I_{q-1}$, with $I_{q-1}$ denoting the interpolation operator by elements of $\mathcal{V}_{q-1}\left(J_{n}\right)$ at the nodes $t^{n-1}+\tau_{i} k_{n}, i=1, \ldots, q, n=1, \ldots, N$, with appropriate $0 \leq \tau_{1}<\cdots<\tau_{q} \leq 1$. It is well known that RK Gauss-Legendre schemes are related to continuous Galerkin methods. A first conclusion, perhaps not observed before, is that all $R K-C$ methods with pairwise distinct nodes in $[0,1]$ can be obtained by applying appropriate numerical quadrature to continuous Galerkin methods. This will be instrumental throughout. It is well known that some RK-C schemes, for instance the RK-Radau IIA methods, exhibit more advantageous stability properties, such as dissipativity, for parabolic equations than the $\mathrm{cG}$ methods. Our association of RK-C methods to cG methods is for convenience and does not affect the stability properties of RK-C (see Example 4.2). 


\subsection{Reconstruction}

Let $R$ be the residual of the approximate solution $U$,

$$
R(t):=U^{\prime}(t)+A U(t)-f(t),
$$

i.e., the amount by which $U$ misses being an exact solution of the differential equation in (1.1) in the linear case, with $B(t, u(t))=f(t)$. Then, the error $e:=u-U$ satisfies the equation

$$
e^{\prime}(t)+A e(t)=-R(t) .
$$

Energy methods applied to (2.5) yield bounds for the error in the norm of $L^{\infty}([0, T] ; H)$ in terms of norms of $R(t)$. However, $R(t)$ is of suboptimal order. In fact, in view of $(2.2)$, the residual can also be written in the form

$$
R(t)=A\left[U(t)-\Pi_{q-1} U(t)\right]-\left[f(t)-\Pi_{q-1} f(t)\right], \quad t \in J_{n} .
$$

This residual is not appropriate for our purposes, since even in the case of a scalar ODE $u^{\prime}(t)=f(t)$ we have $R(t)=-\left[f(t)-\Pi_{q-1} f(t)\right]$, and thus $R(t)$ can only be of order $\mathcal{O}\left(k_{n}^{q}\right)$, although our approximations are piecewise polynomials of degree $q$. In both cases, cG as well as RK-C methods (with nodes satisfying (1.2)), the optimal order of approximation in $L^{\infty}([0, T] ; H)$ is $\mathcal{O}\left(k^{q+1}\right)$. It would thus be desirable to have an error equation with optimal right-hand side. To this end, we introduce a suitable higher order reconstruction $\widehat{U} \in \mathcal{H}_{q+1}$ of the approximation $U$. The function $\widehat{U}$, however, does not provide a better approximation to $u$ than $U$ and its construction and analysis does not require small time steps. We further assume the regularity condition (2.11) on $\widehat{U}$ throughout, and discuss its validity in Section 5 .

The definition of $\widehat{U} \in \mathcal{H}_{q+1}$ is based on appropriate projection operators $\widehat{\Pi}_{q}$ onto $\mathcal{H}_{q}\left(J_{n}\right), n=1, \ldots, N$. To be more precise, we assume that $\Pi_{q-1}$ in (2.2) is associated to $q$ pairwise distinct points $\tau_{1}, \ldots, \tau_{q} \in[0,1]$ with the orthogonality property

$$
\int_{0}^{1} \prod_{i=1}^{q}\left(\tau-\tau_{i}\right) d \tau=0
$$

These points are transformed to the interval $J_{n}$ as $t^{n, i}:=t^{n-1}+\tau_{i} k_{n}$, $i=1, \ldots, q$. Specifically, they are the collocation points for RK-C or the Gauss points for cG. A fundamental property we require for $\widehat{\Pi}_{q}$ is that it agrees with $\Pi_{q-1}$ at $t^{n, i}$ :

$$
\left(\widehat{\Pi}_{q}-\Pi_{q-1}\right) w\left(t^{n, i}\right)=0, \quad i=1, \ldots, q, \quad \forall w \in C\left(J_{n} ; H\right) .
$$


If (2.7) is satisfied, then interpolatory quadrature with abscissae $t^{n, i}$, $i=1, \ldots, q$, integrates polynomials of degree at most $q$ exactly. Therefore, (2.8) leads to the key property of $\widehat{\Pi}_{q}$ that $\left(\widehat{\Pi}_{q}-\Pi_{q-1}\right) w$ is orthogonal to constants in $J_{n}$,

$$
\int_{J_{n}}\left(\widehat{\Pi}_{q}-\Pi_{q-1}\right) w(s) d s=0 \quad \forall w \in C\left(J_{n} ; H\right),
$$

for $n=1, \ldots, N$, which will play a central role in the analysis. For each $n=1, \ldots, N$, we define the reconstruction $\widehat{U} \in \mathcal{H}_{q+1}\left(J_{n}\right)$ of $U$ by

$$
\widehat{U}(t):=U\left(t^{n-1}\right)-\int_{t^{n-1}}^{t} \widehat{\Pi}_{q}[A U(s)-f(s)] d s \quad \forall t \in J_{n} .
$$

Obviously, $\widehat{U}\left(t^{n-1}\right)=U\left(t^{n-1}\right)$. Furthermore, in view of (2.9),

$$
\begin{aligned}
\widehat{U}\left(t^{n}\right) & =U\left(t^{n-1}\right)-\int_{t^{n-1}}^{t^{n}} \widehat{\Pi}_{q}[A U(s)-f(s)] d s \\
& =U\left(t^{n-1}\right)-\int_{t^{n-1}}^{t^{n}} \Pi_{q-1}[A U(s)-f(s)] d s ;
\end{aligned}
$$

taking here relation (2.2) into account, we obtain

$$
\widehat{U}\left(t^{n}\right)=U\left(t^{n-1}\right)+\int_{t^{n-1}}^{t^{n}} U^{\prime}(s) d s=U\left(t^{n}\right),
$$

and conclude that $\widehat{U}$ is continuous in $[0, T]$ and coincides with $U$ at the nodes $t^{n}$. Moreover, we assume throughout that $\widehat{U}$ satisfies the following regularity condition:

$$
\widehat{U}(t) \in V \quad \forall t \in[0, T] .
$$

This property is crucial for the error analysis and entails some minimal regularity of $U^{0}$ and compatibility with $f(0)$, depending on the time-discrete method; see $\S 4.4$. However, $(2.11)$ is always satisfied by fully discrete schemes for evolution PDEs which constitute the most important application of the present framework.

It easily follows from (2.10) that $\widehat{U}$ satisfies the following pointwise equation

$$
\widehat{U}^{\prime}(t)+A U(t)=\widehat{\Pi}_{q} f(t) \quad \forall t \in J_{n} ;
$$

compare with (2.2). In view of (2.12), the residual $\widehat{R}$,

$$
\widehat{R}(t):=\widehat{U}^{\prime}(t)+A \widehat{U}(t)-f(t),
$$


of $\widehat{U}$ can also be written as

$$
\widehat{R}(t)=A[\widehat{U}(t)-U(t)]-\left[f(t)-\widehat{\Pi}_{q} f(t)\right] .
$$

We show in the sequel that $\widehat{R}(t)$ is an a posteriori quantity of the desired order for appropriate choices of $\widehat{\Pi}_{q}$, provided (2.11) is valid.

\subsection{Energy Estimates and Representation of $\widehat{U}-U$}

We let $V:=D\left(A^{1 / 2}\right)$ and denote the norms in $H$ and in $V$ by $|\cdot|$ and $\|\cdot\|$, with $\|v\|:=\left|A^{1 / 2} v\right|=\langle A v, v\rangle^{1 / 2}$, respectively. We identify $H$ with its dual, and let $V^{\star}$ be the topological dual of $V\left(V \subset H \subset V^{\star}\right)$. We still denote by $\langle\cdot, \cdot\rangle$ the duality pairing between $V^{\star}$ and $V$, and by $\|\cdot\|_{\star}$ the dual norm on $V^{\star}$, namely $\|v\|_{\star}:=\left|A^{-1 / 2} v\right|=\left\langle v, A^{-1} v\right\rangle^{1 / 2}$.

We consider, as in $[2,23,24]$, the error functions

$$
e:=u-U \text { and } \hat{e}:=u-\widehat{U} .
$$

Once a suitable reconstruction $\widehat{U}$ of $U$ is in place, the rest of the analysis is rather elementary as the following simple results illustrate; see also [2] for further details. When working with energy estimates the starting point of the analysis is the error equation,

$$
\hat{e}^{\prime}(t)+A \hat{e}(t)=-\widehat{R},
$$

$(\widehat{R}$ is defined in $(2.13),(2.14)$ ) written in its equivalent form

$$
\hat{e}^{\prime}(t)+A e(t)=f-\widehat{\Pi}_{q} f .
$$

The main reason is that working with (2.17) allows the derivation of lower bounds of the error in addition to upper bounds.

Theorem 2.1 (Error estimates) Let the assumptions (2.7) on $\left\{\tau_{i}\right\}_{i=1}^{q}$, (2.8) on $\widehat{\Pi}_{q}$, and (2.11) on $\widehat{U}$ be satisfied. Then the following global upper estimate is valid, for $t \in[0, T]$,

$$
\begin{aligned}
\max _{0 \leq \tau \leq t}\left[|\hat{e}(\tau)|^{2}\right. & \left.+\int_{0}^{\tau}\left(\|e(s)\|^{2}+\frac{1}{2}\|\hat{e}(s)\|^{2}\right) d s\right] \\
& \leq \int_{0}^{t}\|(\widehat{U}-U)(s)\|^{2} d s+2 \int_{0}^{t}\left\|\left(f-\widehat{\Pi}_{q} f\right)(s)\right\|_{\star}^{2} d s .
\end{aligned}
$$

The following local lower estimate is also valid

$$
\frac{1}{3}\|\widehat{U}(t)-U(t)\|^{2} \leq\|e(t)\|^{2}+\frac{1}{2}\|\hat{e}(t)\|^{2} \quad \forall t \in[0, T] .
$$


Proof Multiplying the error equation (2.17) by $\hat{e}(t)$ and using the identity $2\langle A e, \hat{e}\rangle=\|e\|^{2}+\|\hat{e}\|^{2}-\|\widehat{U}-U\|^{2}$, we arrive at

$$
\frac{d}{d t}|\hat{e}(t)|^{2}+\|e\|^{2}+\|\hat{e}\|^{2}=\|\widehat{U}-U\|^{2}+2\left\langle f-\widehat{\Pi}_{q} f, \hat{e}\right\rangle .
$$

This easily leads to the asserted upper bound. Note that we gain control of both $\|e\|$ and $\|\hat{e}\|$, which is crucial for the lower bound. In fact, the latter follows immediately from the triangle inequality.

Remark 2.1 (Optimal Error Estimator) Integrating the local lower bound yields

$$
\begin{aligned}
\frac{1}{3} \int_{0}^{t} \|(\widehat{U} & -U)(s) \|^{2} d s \leq \int_{0}^{t}\left(\|e(s)\|^{2}+\frac{1}{2}\|\hat{e}(s)\|^{2}\right) d s \\
& \leq \int_{0}^{t}\|(\widehat{U}-U)(s)\|^{2} d s+2 \int_{0}^{t}\left\|\left(f-\widehat{\Pi}_{q} f\right)(s)\right\|_{\star}^{2} d s .
\end{aligned}
$$

The estimator $\int_{0}^{t}\|(\widehat{U}-U)(s)\|^{2} d s$ is of optimal order because it is dominated by the integral error $\int_{0}^{t}\left(\|e(s)\|^{2}+\frac{1}{2}\|\hat{e}(s)\|^{2}\right) d s$, which is of order $q+1$. Moreover, the max error $\max _{0 \leq \tau \leq t}|\hat{e}(\tau)|^{2}$ is dominated by the integral error plus data oscillation.

Remark 2.2 (Error Estimate Revised) In case $\widehat{\Pi}_{q} U \neq U$ (cf. Section 5 for cases that this might happen), (2.12) is replaced by

$$
\widehat{U}^{\prime}(t)+A \widehat{\Pi}_{q} U(t)=\widehat{\Pi}_{q} f(t) \quad \forall t \in J_{n},
$$

and (2.17) by

$$
\hat{e}^{\prime}(t)+A e(t)=f-\widehat{\Pi}_{q} f-A\left(U-\widehat{\Pi}_{q} U\right)(t) .
$$

In such a case the estimate of Theorem 2.1 remains valid, provided that a term $2 \int_{0}^{t}\left\|\left(U-\widehat{\Pi}_{q} U\right)(s)\right\|^{2} d s$ is added on the right-hand side.

Even though $\widehat{U}-U$ is computable, we prefer to give a precise characterization.

Theorem 2.2 (Explicit Representation of $\widehat{U}-U$ ) Let

$$
\varphi_{q+1}(x):=(q+1) \int_{0}^{x} \prod_{i=1}^{q}\left(s-\tau_{i}\right) d s .
$$

If $\left\{\tau_{i}\right\}_{i=1}^{q}$ and $\widehat{\Pi}_{q}$ satisfy (2.7) and (2.8), then

$$
\widehat{U}(t)-U(t)=\frac{1}{(q+1) !} k_{n}^{q+1} \widehat{U}^{(q+1)} \varphi_{q+1}\left(\frac{t-t^{n-1}}{k_{n}}\right) .
$$


In addition, if $\widehat{U}$ satisfies (2.11) and

$\alpha_{q}:=\frac{1}{[(q+1) !]^{2}} \int_{0}^{1}\left[\varphi_{q+1}(x)\right]^{2} d x$ and $\beta_{q}:=\frac{1}{(q+1) !} \max _{0 \leq x \leq 1}\left|\varphi_{q+1}(x)\right|$,

then

$$
\begin{gathered}
\int_{J_{n}}\|(\widehat{U}-U)(t)\|^{2} d s=\alpha_{q} k_{n}^{2 q+3}\left\|\widehat{U}^{(q+1)}\right\|^{2}, \\
\max _{i \in J_{n}}|(\widehat{U}-U)(t)|=\beta_{q} k_{n}^{q+1}\left|\widehat{U}^{(q+1)}\right| .
\end{gathered}
$$

Proof Subtracting (2.2) from (2.12), we obtain

$$
\widehat{U}^{\prime}-U^{\prime}=\left(\widehat{\Pi}_{q}-\Pi_{q-1}\right)(f-A U),
$$

whence, in view of $(2.8)$,

$$
\left(\widehat{U}^{\prime}-U^{\prime}\right)\left(t^{n, i}\right)=0, \quad i=1, \ldots, q .
$$

Therefore, we deduce

$$
\left(\widehat{U}^{\prime}-U^{\prime}\right)(t)=\frac{1}{q !} \widehat{U}^{(q+1)} k_{n}^{q} \prod_{i=1}^{q}\left(\frac{t-t^{n-1}}{k_{n}}-\tau_{i}\right) .
$$

Relation (2.21) follows immediately upon integration of (2.25). The asserted estimates follow from the change of variables $x=(t-$ $\left.t^{n-1}\right) / k_{n}$ to $[0,1]$.

Remark 2.3 (Computable Error Estimator) Regardless of the norm, the (properly scaled) quantity $\widehat{U}^{(q+1)}=A U^{(q)}+\left(\widehat{\Pi}_{q} f\right)^{(q)}$ is what dictates the local size of the estimator. Note that $\widehat{U}^{(q+1)}$ is easily computable.

Corollary 2.1 (Explicit Error Estimates) If (2.7), (2.8), and (2.11) are valid, then the following lower and upper bounds hold

$$
\begin{aligned}
& \frac{\alpha_{q}}{3} \sum_{n=1}^{m} k_{n}^{2 q+3}\left\|\widehat{U}^{(q+1)}\right\|^{2} \\
& \quad \leq \max _{0 \leq \tau \leq t^{m}}\left[|\hat{e}(\tau)|^{2}+\int_{0}^{\tau}\left(\|e(s)\|^{2}+\frac{1}{2}\|\hat{e}(s)\|^{2}\right) d s\right] \\
& \quad \leq \alpha_{q} \sum_{n=1}^{m} k_{n}^{2 q+3}\left\|\widehat{U}^{(q+1)}\right\|^{2}+2 \int_{0}^{t^{m}}\left\|\left(f-\widehat{\Pi}_{q} f\right)(s)\right\|_{\star}^{2} d s .
\end{aligned}
$$

Proof Combine Theorems 2.1 and 2.2 with (2.18). 
Remark 2.4 (A Priori Estimates) We stress that if $f$ is a piecewise polynomial of degree at most $q$, then the data oscillation term above vanishes. Otherwise, we observe that all terms above appear to be of the same order, namely $\mathcal{O}\left(k_{n}^{2 q+3}\right)$ locally, which is consistent with the global order $q+1$ of the methods considered in this paper, as we will see later. If $f=0$, and $\widehat{U}^{(q+1)}$ converges to $u^{(q+1)}$, then we could formally write

$$
\sum_{n=1}^{m} k_{n}^{2 q+3}\left\|\widehat{U}^{(q+1)}\right\|^{2} \approx \int_{0}^{t^{m}} k(t)^{2 q+2}\left\|u^{(q+1)}(t)\right\|^{2} d t,
$$

where $k(t)$ stands for the piecewise constant time-step function. This is consistent with the a priori error representation formula.

\section{The Continuous Galerkin Method}

In this section we first recall the continuous Galerkin method (cG). In $\S 3.1$ we cast $\mathrm{cG}$ within the abstract framework of $\S 1$, and so that both Theorems 2.1 and 2.2 apply to cG. We then extend the theory to nonlinear equations of the form (1.1) in $\S 3.2$. A posteriori estimates for $\mathrm{cG}$ for ODEs are established in [12].

The cG approximation $U$ to the solution $u$ of (1.1) is defined as follows: We seek $U \in \mathcal{V}_{q}$ such that $U(0)=u^{0}$ and

$$
\int_{J_{n}}\left[\left\langle U^{\prime}, v\right\rangle+\langle A U, v\rangle\right] d t=\int_{J_{n}}\langle f, v\rangle d t \quad \forall v \in \mathcal{V}_{q-1}\left(J_{n}\right)
$$

for $n=1, \ldots, N$. For local uniqueness and existence results for $\mathrm{cG}$ as well as for a priori error estimates, including nonlinear parabolic equations, we refer to [1,4]. It follows from (3.1) that $U \in \mathcal{V}_{q}$ satisfies also the following pointwise equation

$$
U^{\prime}(t)+P_{q-1} A U(t)=P_{q-1} f(t) \quad \forall t \in J_{n},
$$

with $P_{\ell}$ denoting the (local) $L^{2}$ orthogonal projection operator onto $\mathcal{H}_{\ell}\left(J_{n}\right)$ :

$$
\int_{J_{n}}\left\langle P_{\ell} w, v\right\rangle d s=\int_{J_{n}}\langle w, v\rangle d s \quad \forall v \in \mathcal{H}_{\ell}\left(J_{n}\right)
$$

We thus conclude that $\mathrm{cG}$ is indeed a particular case, with $\Pi_{q-1}=$ $P_{q-1}$, of the general class of methods described by $(2.2)$. 


\subsection{Continuous Galerkin Reconstruction}

We let $\widehat{\Pi}_{q}:=P_{q}$ and define the $c G$ reconstruction $\widehat{U} \in \mathcal{H}_{q+1}\left(J_{n}\right)$ via (2.10). This expression reads pointwise

$$
\widehat{U}^{\prime}(t)+A U(t)=P_{q} f(t) \quad \forall t \in J_{n} .
$$

We need now to identify the nodes $\left\{\tau_{i}\right\}_{i=1}^{q}$ in (2.7). Let $p_{0}, p_{1}, \ldots$ be the Legendre polynomials shifted to $J_{n}$ and normalized. Since

$$
\left(P_{q}-P_{q-1}\right) w=\int_{J_{n}} w(s) p_{q}(s) d s \cdot p_{q} \quad \forall w \in L^{2}\left(J_{n}\right),
$$

we infer from (2.8) that $\left\{t^{n, i}\right\}_{i=1}^{q}$ are the zeros of $p_{q}$ and thus $\left\{\tau_{i}\right\}_{i=1}^{q}$ are the Gauss points in $(0,1)$.

A consequence of $(2.24)$ is that $\left(\widehat{U}^{\prime}-U^{\prime}\right)\left(t^{n, i}\right)=0$ for $t^{n, i}=$ $t^{n-1}+\tau_{i} k_{n}$. In this case we can also identify the zeros of $\widehat{U}-U$. In fact, since

$$
\left(\widehat{U}^{\prime}-U^{\prime}\right)(t)=\frac{1}{q !} k_{n}^{q} \widehat{U}^{(q+1)} \prod_{i=1}^{q}\left(\frac{t-t^{n-1}}{k_{n}}-\tau_{i}\right)
$$

by virtue of (2.25), and $\widehat{U}-U$ vanishes at $t^{n-1}$ and $t^{n}$, the zeros of $\widehat{U}-U$ are the $q+1$ Lobatto points in $J_{n}$, namely the roots of $\varphi_{q+1}$ in $(2.21)$; see [8, p. 104].

Remark 3.1 (Variational Conditions for $\widehat{U}$ ) Upon subtracting (3.2) from (3.3) we get the following characterization of the $\mathrm{cG}$ reconstruction $\widehat{U} \in \mathcal{H}_{q+1}$ :

$$
\left\{\begin{array}{l}
\widehat{U}\left(t^{n-1}\right)=U\left(t^{n-1}\right), \quad \widehat{U}\left(t^{n}\right)=U\left(t^{n}\right), \\
\int_{J_{n}}\left(\widehat{U}^{\prime}-U^{\prime}, v\right) d t=0 \quad \forall v \in \mathcal{V}_{q-1}\left(J_{n}\right) .
\end{array}\right.
$$

Remark 3.2(A Priori Projection and Elliptic Reconstruction) In the derivation of optimal order a priori error estimates the function $W \in \mathcal{V}_{q}\left(J_{n}\right)$ defined by

$$
\left\{\begin{array}{l}
W\left(t^{n-1}\right)=u\left(t^{n-1}\right), \quad W\left(t^{n}\right)=u\left(t^{n}\right), \\
\int_{J_{n}}\left(u^{\prime}-W^{\prime}, v\right) d t=0 \quad \forall v \in \mathcal{V}_{q-1}\left(J_{n}\right)
\end{array}\right.
$$

plays a fundamental role $[1,4,27]$, analogous to the role of the elliptic projection of the exact solution in the derivation of optimal order a priori error estimates for space discrete finite element methods for 
parabolic equations [27]. The continuous Galerkin reconstruction $\widehat{U} \in$ $\mathcal{H}_{q+1}$ 'solves' problem (3.4) that is in a sense 'dual' to (3.5). Note the similarity to the relation between the elliptic projection and the elliptic reconstruction of [22] in the a posteriori error analysis of space discrete finite element methods for parabolic equations.

We recall that Theorem 2.2 provides a simple representation for $\widehat{U}-U$ for cG. We may wonder about the lower order case $q=1$ and consistency with [2]; this is discussed next.

Remark 3.3 (Case $q=1$ : The Crank-Nicolson-Galerkin Method) Since $\tau_{1}=\frac{1}{2}$ and the Lobatto points in $J_{n}$ are just $t^{n-1}$ and $t^{n},(2.24)$ and (2.25) yield for all $t \in J_{n}$

$\widehat{U}(t)-U(t)=\int_{t^{n-1}}^{t}\left(P_{1}-P_{0}\right)(f-A U)(s) d s=\frac{1}{2} \widehat{U}^{\prime \prime}\left(t-t^{n-1}\right)\left(t-t^{n}\right)$.

We now derive a different, but equivalent, representation of $\widehat{U}^{\prime \prime}$. We note that $U$ being linear implies

$$
\left(P_{1}-P_{0}\right) A U(t)=p_{1}(t) \int_{J_{n}} A U(s) p_{1}(s) d s,
$$

where $p_{1}(t)=\sqrt{\frac{12}{k_{n}^{3}}}\left(t-t^{n-\frac{1}{2}}\right)$ is the second orthonormal Legendre polynomial in $J_{n}$. Since $p_{1}$ is orthogonal to constants, we see that

$$
\int_{J_{n}} A U(s) p_{1}(s) d s=A U^{\prime} \int_{J_{n}} s p_{1}(s) d s=\sqrt{\frac{k_{n}^{3}}{12}} A U^{\prime} .
$$

On the other hand, we have

$$
\left(P_{1}-P_{0}\right) f(t)=p_{1}(t) \int_{J_{n}} f(s) p_{1}(s) d s
$$

Integrating in time from $t^{n-1}$ to $t$, we end up with the expression

$\widehat{U}(t)-U(t)=\left(t-t^{n-1}\right)\left(t-t^{n}\right)\left(-\frac{1}{2} A U^{\prime}+\frac{6}{k_{n}^{3}} \int_{J_{n}} f(s)\left(s-t^{n-\frac{1}{2}}\right) d s\right)$,

which turns out to be the relation (3.4) in [2]. This shows that Theorem 2.2 extends [2] for any $q>1$. 


\subsection{Continuous Galerkin Method for Nonlinear Equations}

In this subsection we consider the discretization of (1.1). We assume that $B(t, \cdot)$ can be extended to an operator from $V$ into $V^{\star}$. A natural condition for (1.1) to be locally of parabolic type is the following local one-sided Lipschitz condition

$$
\langle B(t, v)-B(t, w), v-w\rangle \leq \lambda\|v-w\|^{2}+\mu|v-w|^{2} \quad \forall v, w \in T_{u}
$$

in a tube $T_{u}:=\left\{v \in V: \min _{t}\|u(t)-v\| \leq 1\right\}$, around the solution $u$, uniformly in $t$, with constants $\lambda<1$ and $\mu \geq 0$. If $F(t, v):=$ $A v-B(t, v)$, then it turns out that (3.6) can be written in the form of a Gårding-type inequality,

$$
\langle F(t, v)-F(t, w), v-w\rangle \geq(1-\lambda)\|v-w\|^{2}-\mu|v-w|^{2}
$$

for $v, w \in T_{u}$. Furthermore, in order to ensure that an appropriate residual is of the correct order, we make use of the following local Lipschitz condition for $B(t, \cdot)$

$$
\|B(t, v)-B(t, w)\|_{\star} \leq L\|v-w\| \quad \forall v, w \in T_{u}
$$

with a constant $L$, not necessarily less than one.

The tube $T_{u}$ is here defined in terms of the norm of $V$ for concreteness. The analysis may be modified to yield a posteriori error estimates under conditions analogous to (3.6) and (3.8) for $v$ and $w$ belonging to tubes defined in terms of other norms, not necessarily the same for both arguments.

We recall that $\mathrm{cG}$ for (1.1) consists of seeking a function $U$ : $[0, T] \rightarrow V$, continuous and piecewise polynomial of degree at most $q$, such that $U(0)=u(0)$ and

$$
\int_{J_{n}}\left[\left\langle U^{\prime}, v\right\rangle+\langle A U, v\rangle\right] d t=\int_{J_{n}}\langle B(t, U), v\rangle d t \quad \forall v \in \mathcal{V}_{q-1}\left(J_{n}\right)
$$

for $n=1, \ldots, N$. The $\mathrm{cG}$ approximation $U$ satisfies the pointwise equation

$$
U^{\prime}(t)+P_{q-1} A U(t)=P_{q-1} B(t, U(t)) \quad \forall t \in J_{n} .
$$

For existence and local uniqueness results for the continuous Galerkin approximations as well as for a priori error estimates we refer to [1].

The continuous Galerkin reconstruction $\widehat{U} \in \mathcal{H}_{q+1}\left(J_{n}\right)$ is now defined by

$$
\widehat{U}(t):=U\left(t^{n-1}\right)-\int_{t^{n-1}}^{t}\left[A U(s)-P_{q} B(s, U(s))\right] d s \quad \forall t \in J_{n}
$$


this extends (2.10) with $\widehat{\Pi}_{q}=P_{q}$. Obviously, $\widehat{U}$ satisfies the pointwise equation

$$
\widehat{U}^{\prime}(t)+A U(t)=P_{q} B(t, U(t)) \quad \forall t \in J_{n} .
$$

Remark 3.4 (Conditional Estimates) The following estimates are valid under the assumption that $U(t), \widehat{U}(t) \in T_{u}$, for all $t \in[0, T]$. This restrictive assumption can sometimes be verified a posteriori. In such cases, the final estimate holds subject to a condition that $U$ or $\widehat{U}$ may or may not satisfy but can be computationally verified. The derivation of these bounds requires the use of fine properties of the specific underlying PDE, as was done in [18,23], and therefore goes beyond the scope of the present paper.

Theorem 3.1 (Error Estimates for Nonlinear Equations) Assume that $U(t), \widehat{U}(t) \in T_{u}$, for all $t \in[0, T]$. Then, the following upper bound is valid, for any $\varepsilon \in\left(0, \frac{1}{2}(1-\lambda)\right)$,

$$
\begin{gathered}
\max _{0 \leq \tau \leq t}\left[|\hat{e}(\tau)|^{2}+(1-\lambda-2 \varepsilon) \int_{0}^{\tau} \mathrm{e}^{3 \mu(\tau-s)}\left(\|\hat{e}(s)\|^{2}+\|e(s)\|^{2}\right) d s\right] \\
\leq \int_{0}^{t} \mathrm{e}^{3 \mu(t-s)}\left[2 \mu|(\widehat{U}-U)(s)|^{2}+\left(\frac{L^{2}}{2 \varepsilon}+1\right)\|(\widehat{U}-U)(s)\|^{2}\right. \\
\left.+\frac{1}{\varepsilon}\left\|R_{U}(s)\right\|_{\star}^{2}\right] d s .
\end{gathered}
$$

Proof Subtracting (3.12) from the differential equation in (1.1), we obtain

$$
\hat{e}^{\prime}(t)+A e(t)=B(t, u(t))-B(t, U(t))+R_{U}(t)
$$

with

$$
R_{U}(t)=B(t, U(t))-P_{q} B(t, U(t))
$$

compare with (6.19) and (6.20) in [2]. Proceeding as in [2], namely taking the inner product with $\hat{e}(t)$, from (3.13) we can establish the desired upper bound.

Let us note that the lower bound in Theorem 2.1 is obviously also valid in the nonlinear case. 


\section{Runge-Kutta Collocation Methods}

Let $q \in \mathbb{N}$ and $\tau_{1}, \ldots, \tau_{q} \in[0,1]$ be pairwise different, $0 \leq \tau_{1}<\cdots<$ $\tau_{q} \leq 1$. We recall that $0=t^{0}<t^{1}<\cdots<t^{N}=T$ is a partition of $[0, T], J_{n}=\left(t^{n-1}, t^{n}\right]$ and $k_{n}=t^{n}-t^{n-1}$, and set $t^{n, i}:=t^{n-1}+\tau_{i} k_{n}$. The collocation method with nodes $\tau_{1}, \ldots, \tau_{q}$, applied to (1.1), reads: We seek $U \in \mathcal{V}_{q}$ such that

$$
U^{\prime}\left(t^{n, i}\right)+F\left(t^{n, i}, U\left(t^{n, i}\right)\right)=0, \quad i=1, \ldots, q,
$$

for $n=1, \ldots, N$; here $U(0)=u(0)$. We do not consider linear equations separately.

\subsection{Runge-Kutta and Collocation Methods}

For $q \in \mathbb{N}$, a $q$-stage Runge-Kutta (RK) method is described by the constants $a_{i j}, b_{i}, \tau_{i}, i, j=1, \ldots, q$, arranged in a Butcher tableau,

$$
\begin{array}{ccc|c}
a_{11} & \ldots & a_{1 q} & \tau_{1} \\
\vdots & & \vdots & \vdots \\
a_{q 1} & \ldots & a_{q q} & \tau_{q} \\
\hline b_{1} & \ldots & b_{q} &
\end{array} .
$$

Given an approximation $U^{n-1}$ to $u\left(t^{n-1}\right)$, the $n$-th step of the Runge-Kutta method applied to (1.1) that yields the approximation $U^{n}$ to $u\left(t^{n}\right)$ is

$$
\left\{\begin{aligned}
U^{n, i} & =U^{n-1}-k_{n} \sum_{j=1}^{q} a_{i j} F\left(t^{n, j}, U^{n, j}\right), \quad i=1, \ldots, q, \\
U^{n} & =U^{n-1}-k_{n} \sum_{i=1}^{q} b_{i} F\left(t^{n, i}, U^{n, i}\right) ;
\end{aligned}\right.
$$

here $U^{n, i}$ are the intermediate stages, which are approximations to $u\left(t^{n, i}\right)$.

Let $r$ and $s$ be the largest integers such that

$$
\begin{aligned}
& \sum_{i=1}^{q} b_{i} \tau_{i}^{\ell}=\frac{1}{\ell+1}, \quad \ell=0, \ldots, s-1, \\
& \sum_{j=1}^{q} a_{i j} \tau_{j}^{\ell}=\frac{\tau_{i}^{\ell+1}}{\ell+1}, \quad \ell=0, \ldots, r-1, \quad i=1, \ldots, q .
\end{aligned}
$$


The stage order of the Runge-Kutta method is $p^{\prime}:=\min (s, r)$. The classical (nonstiff) order of the method is the largest integer $p$ such that after one step of the RK method, with $y^{n-1}:=y\left(t^{n-1}\right)$, there holds $y\left(t^{n}\right)-y^{n}=O\left(k_{n}^{p+1}\right)$ for smooth solutions $y$ of ODEs with bounded derivatives; $p$ is the superorder of RK.

The collocation method (4.1) is equivalent to the Runge-Kutta method with

$$
a_{i j}:=\int_{0}^{\tau_{i}} L_{j}(\tau) d \tau, \quad b_{i}:=\int_{0}^{1} L_{i}(\tau) d \tau, \quad i, j=1, \ldots, q,
$$

with $L_{1}, \ldots, L_{q}$ the Lagrange polynomials of degree $q-1$ associated with the nodes $\tau_{1}, \ldots, \tau_{q}$, in the sense that $U\left(t^{n, i}\right)=U^{n, i}, i=$ $1, \ldots, q$, and $U\left(t^{n}\right)=U^{n}$; see [15, Theorem 7.6]. This is the RungeKutta Collocation $(R K-C)$ class and satisfies $(2.2)$ with $\Pi_{q-1}=I_{q-1}$. Conversely, a $q$-stage Runge-Kutta method with pairwise different $\tau_{1}, \ldots, \tau_{q}$ is equivalent to the collocation method with the same nodes, if and only if its stage order is at least $q$; see [15, Theorem 7.7]. Given the stages $U^{n, i}, i=1, \ldots, q$, of the Runge-Kutta method, the collocation approximation $U \in \mathcal{V}_{q}\left(J_{n}\right)$ is recovered by interpolating $\left(t^{n, i}, U^{n, i}\right), i=1, \ldots, q$, and either $\left(t^{n-1}, U^{n-1}\right)$, if $\tau_{1}>0$, or $\left(t^{n}, U^{n}\right)$, if $\tau_{q}<1$. In case $\tau_{1}=0$ and $\tau_{q}=1$, the collocation approximation is continuously differentiable; therefore, for instance, if $\tau_{1}=0, U$ can be recovered by interpolating $\left(t^{n-1}, U^{n-1}\right)$ and $\left(t^{n, i}, U^{n, i}\right), i=1, \ldots, q$, and requiring $U^{\prime}\left(t^{n-1}\right)=-F\left(t^{n-1}, U^{n-1}\right)$. Most of the important RK methods belong to the RK-C class.

\subsection{Order in $L^{\infty}([0, T] ; H)$ and Superorder.}

In this work we focus on estimators for the $L^{\infty}([0, T] ; H)$ norm of the error. If $U$ is piecewise polynomial of degree $q$, then the highest possible order of convergence in $L^{\infty}([0, T] ; H)$ is $q+1$, namely

$$
\max _{0 \leq t \leq T}|u(t)-U(t)|=O\left(k^{q+1}\right)
$$

with $k:=\max _{n} k_{n}$. This fact follows from basic approximation theory results and is valid even in the case of initial value problems for ODEs with smooth solutions. For the optimal order of convergence $q+1$ in (4.3) to be attained, a condition on the RK-C method is required, as the case $q=1, \tau_{1}=1$ shows, which reduces to the backward Euler method, a scheme yielding first order approximations even at the nodes $t^{1}, \ldots, t^{N}$. This is related to the convergence order at the nodes $\left\{t^{n}\right\}_{n=1}^{N}$. Indeed, it is well known that the classical order of the 
RK-C method is $p>q$, if and only if the nodes $\tau_{1}, \ldots, \tau_{q}$ satisfy the orthogonality condition

$$
\int_{0}^{1} \prod_{i=1}^{q}\left(\tau-\tau_{i}\right) v(\tau) d \tau=0 \quad \forall v \in \mathbb{P}_{r}
$$

for $r \geq 1$, where $r=p-q-1,[15$, Theorem 7.8]. We recall that condition (4.4) is obviously satisfied if and only if every element of $\mathbb{P}_{q+r}$ and its Lagrange interpolant at $\tau_{1}, \ldots, \tau_{q}$ have the same integral in $[0,1]$, i.e., if the interpolatory quadrature formula with abscissae $\tau_{1}, \ldots, \tau_{q}$ integrates the elements of $\mathbb{P}_{q+r}=\mathbb{P}_{p-1}$ exactly.

A necessary and sufficient condition for (4.3) to hold for problems with smooth solutions is that $p \geq q+1$, that is (2.7) is satisfied. In the sequel we assume therefore that (2.7) (and hence (4.3)) holds and we will establish a posteriori estimates of this order in $L^{\infty}([0, T] ; H)$.

It is worth noting though, that there exist interesting methods where (2.7) fails to be valid, and thus the order in $L^{\infty}([0, T] ; H)$ is $q$. For such methods, in general, the residual (2.6) is not suboptimal any longer and thus estimates based on the original formulation of the method and its error equation (2.5) are possible. Examples of such methods are the Backward Euler Method (see Example 4.2), and the Trapezoidal Method (see Example 4.3). For these methods however it might be preferable to consider alternative formulations involving lower polynomial degrees for $U$ than those corresponding to their RK-C formulation. Such approaches are discussed in [24] for the Backward Euler Method and in Example 4.7 for the Trapezoidal Method.

Next, we briefly examine the case $p>q+1$. Although the order of the method in $L^{\infty}([0, T] ; H)$ is provided by (4.3), the classical order $p$ of the RK-C method corresponds to the superconvergence order at the nodes $\left\{t^{n}\right\}_{n=1}^{N}$ (superorder for short) of the discrete solution $U$ of initial value problems for ODEs with smooth solutions; this is the standard terminology of RK methods $[15,16]$. Since the seminal work of Crouzeix [6] it is known that the superorder is limited for linear problems unless nontrivial compatibility conditions of the form

$$
f \in D\left(A^{\rho}\right), \quad U^{0} \in D\left(A^{\rho+1}\right)
$$

are valid for $1 \leq \rho \leq q-1$. A requirement such as (4.5) may fail to be fulfilled in practice $[20,21,27]$. This lack of nodal superconvergence is usually called order reduction [27]. In [3] we derive a posteriori error bounds that account for nodal superconvergence by implicitly assuming compatibility conditions of the type (4.5). 
We now recall three important classes of collocation methods.

Example 4.1 (RK Gauss-Legendre) Let $\tau_{1}, \ldots, \tau_{q}$ be the zeros of the Legendre polynomial of degree $q$, shifted to $(0,1)$. Then the superorder $p$ of the collocation method is $p=2 q$. The collocation method is equivalent to the $q$-stage RK Gauss-Legendre method; the latter has stage order $q$ and is $B$-stable. The first member of the family of RK Gauss-Legendre methods, i.e., $q=1$, is the Crank-Nicolson scheme.

Example 4.2 (RK Radau IIA) Let $\tau_{1}, \ldots, \tau_{q} \in(0,1]$ be the abscissae of the Radau quadrature formula, with $\tau_{q}=1$. Then the superorder $p$ of the collocation method is $p=2 q-1$. The collocation method is equivalent to the $q$-stage RK Radau IIA method; the latter has stage order $q$ and is $B$-stable and strongly $A$-stable. The first member of the family of RK Radau IIA methods is the backward Euler scheme $(q=1)$ which, however, does not satisfy (1.2) but is examined in [24].

Example 4.3 (RK Lobatto IIIA) Let $0=\tau_{1}<\tau_{2}<\cdots<\tau_{q}=1$ be the abscissae of the Lobatto quadrature formula. The collocation method has a superorder $p=2 q-2$ and is equivalent to the $q$-stage Runge-Kutta-Lobatto IIIA method; the latter has stage order $q$, is $A$-stable but it is not $B$-stable. The first member of the family of RK Lobatto IIIA methods is the trapezoidal scheme $(q=2)$ which, however, does not satisfy (1.2) (see Example 4.7).

\subsection{Pointwise Equation and Residual}

To establish a posteriori error estimates we first derive a pointwise equation for the RK-C approximation $U$. To this end we introduce an interpolation operator $I_{q-1}$, for continuous functions $v$ defined on $J_{n}$,

$$
I_{q-1} v \in \mathcal{H}_{q-1}\left(J_{n}\right): \quad\left(I_{q-1} v\right)\left(t^{n, i}\right)=v\left(t^{n, i}\right), \quad i=1, \ldots, q .
$$

It turns out that (4.1) can be equivalently written in the form

$$
U^{\prime}(t)=-I_{q-1} F(t, U(t))=-A I_{q-1} U(t)+I_{q-1} B(t, U(t)),
$$

$t \in J_{n}$. Note that, in the linear case, (4.7) is a particular case of (2.2) with $\Pi_{q-1}=I_{q-1}$.

Let now $R$ denote the residual of the RK-C approximation, $R(t):=$ $U^{\prime}(t)+F(t, U(t))$. In view of $(4.7), R(t)$ can be rewritten in the form

$$
R(t)=F(t, U(t))-I_{q-1} F(t, U(t)) \quad \forall t \in J_{n} .
$$


This residual is in general of order $O\left(k^{q}\right)$, since it is the error of the interpolation by piecewise polynomials of degree at most $q-1$. This order suffices only if the order of the method is also q, i.e., if (2.7) is not satisfied. This is the case of the backward Euler method (see [24]) and the trapezoidal method (see Example 4.7).

Since we assume that $(2.7)$ is satisfied, and so $U(t)$ is an approximation of order $q+1$ to $u(t)$, for all $t$, then $R(t)$ is of suboptimal order; this is consistent with (2.6). To recover the optimal order $q+1$, we will next introduce a $R K-C$ reconstruction $\widehat{U}$ of the approximation $U$.

\subsection{RK-C Reconstruction}

Let $t^{n, 0} \neq t^{n, i}$, for $i=1, \ldots, q$, be an extra point, which may or may not belong to $J_{n}$. We define the extended interpolation operator $\widehat{I}_{q}$ by

$$
\widehat{I}_{q} v \in \mathcal{H}_{q}\left(J_{n}\right): \quad\left(\widehat{I}_{q} v\right)\left(t^{n, i}\right)=v\left(t^{n, i}\right), \quad i=0,1, \ldots, q,
$$

for all continuous functions $v$ on $[0, T]$. Since $\widehat{I}_{q}$ satisfies $(2.8)$ by definition, it also satisfies the orthogonality property (2.9).

We now define a $R K-C$ reconstruction $\widehat{U} \in \mathcal{H}_{q+1}\left(J_{n}\right)$ of the approximation $U$ by

$$
\widehat{U}(t):=U\left(t^{n-1}\right)-\int_{t^{n-1}}^{t} \widehat{I}_{q} F(s, U(s)) d s \quad \forall t \in J_{n} .
$$

As a consequence of (2.9), and the discussion following (2.10), $\widehat{U}$ is continuous on $[0, T]$. Moreover, by differentiation of $\widehat{U}(t)$ and definition of $F(t, U)$, we deduce

$$
\widehat{U}^{\prime}(t)=-\widehat{I}_{q} F(t, U(t))=-\widehat{I}_{q} A U(t)+\widehat{I}_{q} B(t, U(t)) \quad \forall t \in J_{n}
$$

If $t^{n, 0} \in J_{n}$, then $\widehat{I}_{q} U=U$ and (4.11) becomes the following counterpart of (3.12)

$$
\widehat{U}^{\prime}(t)+A U(t)=\widehat{I}_{q} B(t, U(t)) \quad \forall t \in J_{n} .
$$

Example 4.4 (RK Radau IIA) According to Example 4.2, let $q>1$, $\tau_{q}=1$ and $\tau_{1}>0$. A natural choice for $\tau_{0}$ is $\tau_{0}=0$, i.e., $t^{n, 0}=t^{n-1}$, for which the resulting reconstruction $\widehat{U}$ is continuously differentiable. Indeed, (4.12) yields

$$
\widehat{U}^{\prime}\left(t^{n-}\right)=-A U^{n}+B\left(t^{n}, U^{n}\right) .
$$


Using again (4.12), this time in the interval $J_{n+1}$, and choosing $t^{n+1,0}=$ $t^{n}$, we get

$$
\widehat{U}^{\prime}\left(t^{n+}\right)=-A U^{n}+B\left(t^{n}, U^{n}\right) ;
$$

consequently, $\widehat{U}$ is differentiable at the node $t^{n}$. On the other hand, the time reconstruction introduced by Makridakis and Nochetto for RK Radau IIA methods in the context of dG is just continuous [23, Lemma 2.1]. This is due to the fact that the present time reconstruction is one-degree higher than that in [23] for the same $q$. In [3] we further investigate the relation between $\mathrm{dG}$ and $\mathrm{cG}$ formulations and their corresponding reconstructions and present a unified formulation that covers all schemes, namely, cG, dG, RK-C and perturbed collocation methods.

Example 4.5 (The Crank-Nicolson Method: Two-Point Estimator.) Let $q=1, \tau=1 / 2$ and set $t^{n-\frac{1}{2}}:=\left(t^{n-1}+t^{n}\right) / 2=t^{n-1}+k_{n} / 2$. The RK-C method (4.1) reads: seek $U \in \mathcal{V}_{1}$ such that

$$
U^{\prime}\left(t^{n-\frac{1}{2}}\right)+A U\left(t^{n-\frac{1}{2}}\right)=B\left(t^{n-\frac{1}{2}}, U\left(t^{n-\frac{1}{2}}\right)\right), \quad n=1, \ldots, N
$$

with $U(0)=u(0)$. Now, since

$$
U^{\prime}\left(t^{n-\frac{1}{2}}\right)=\bar{\partial} U^{n}:=\frac{U^{n}-U^{n-1}}{k_{n}}, \quad U\left(t^{n-\frac{1}{2}}\right)=U^{n-\frac{1}{2}}:=\frac{U^{n-1}+U^{n}}{2},
$$

the scheme (4.13) can also be written in the standard Crank-Nicolson form

$$
\bar{\partial} U^{n}+A U^{n-\frac{1}{2}}=B\left(t^{n-\frac{1}{2}}, U^{n-\frac{1}{2}}\right), \quad n=1, \ldots, N,
$$

with $U^{0}=u(0)$. Conversely, if the Crank-Nicolson approximations $\left\{U^{n}\right\}_{n=1}^{N}$ are given by (4.14), then the RK-C approximation $U \in$ $\mathcal{V}_{1}$ is recovered by linearly interpolating between these nodal values. Now let $\tau_{0} \in[0,1], \tau_{0} \neq 1 / 2$, and $t^{n, 0}:=t^{n-1}+\tau_{0} k_{n}$. Then, the interpolation operator $\widehat{I}_{1}$ is given on $J_{n}$ by $\widehat{I}_{1} v \in \mathcal{H}_{1}\left(J_{n}\right)$ such that $\left(\widehat{I}_{1} v\right)\left(t^{n-\frac{1}{2}}\right)=v\left(t^{n-\frac{1}{2}}\right)$ and $\left(\widehat{I}_{1} v\right)\left(t^{n, 0}\right)=v\left(t^{n, 0}\right)$. In particular, for $\tau_{0}=0$,

$$
\begin{aligned}
\left(\widehat{I}_{1} B(\cdot, U)\right)(t) & =B\left(t^{n-\frac{1}{2}}, U^{n-\frac{1}{2}}\right) \\
& +\frac{2\left(t-t^{n-\frac{1}{2}}\right)}{k_{n}}\left[B\left(t^{n-\frac{1}{2}}, U^{n-\frac{1}{2}}\right)-B\left(t^{n-1}, U^{n-1}\right)\right] .
\end{aligned}
$$

Therefore

$$
\widehat{U}(t)=U^{n-1}-\int_{t^{n-1}}^{t} A U(s) d s+\int_{t^{n-1}}^{t}\left(\widehat{I}_{1} B(\cdot, U)\right)(s) d s ;
$$


this coincides with (6.14) in [2]. Moreover, the estimator $\widehat{U}-U$ is controlled by

$$
\widehat{U}^{\prime \prime}=-A \frac{U^{n}-U^{n-1}}{k_{n}}+\frac{2}{k_{n}}\left[B\left(t^{n-\frac{1}{2}}, U^{n-\frac{1}{2}}\right)-B\left(t^{n-1}, U^{n-1}\right)\right]
$$

according to Theorem 2.2.

Example 4.6 (The Crank-Nicolson Method: Three-Point Estimator.) Consider the Crank-Nicolson method, but now choose $t^{n, 0}$ to be the collocation point in the previous interval for $n>1$, i.e., $t^{n, 0}=$ $t^{n-1,1}=t^{n-\frac{3}{2}}$, and in the next interval for $n=1$. Then key properties $F\left(t^{n, 0}, U\left(t^{n, 0}\right)\right) \in V$ and (4.9) of the reconstruction remain valid. Furthermore, according to Theorem 2.2, the estimator hinges on $\widehat{U}^{\prime \prime}$, namely

$$
\widehat{U}^{\prime \prime}=-\frac{2}{k_{n}+k_{n-1}}\left(F\left(t^{n-\frac{1}{2}}, U^{n-\frac{1}{2}}\right)-F\left(t^{n-\frac{3}{2}}, U^{n-\frac{3}{2}}\right)\right) .
$$

Invoking (4.2), namely $U^{n}-U^{n-1}=-k_{n} F\left(t^{n-\frac{1}{2}}, U^{n-\frac{1}{2}}\right)$, yields the estimator

$$
\widehat{U}^{\prime \prime}=\frac{2}{k_{n}+k_{n-1}}\left(\frac{U^{n}-U^{n-1}}{k_{n}}-\frac{U^{n-1}-U^{n-2}}{k_{n-1}}\right),
$$

regardless of whether $F(t, U)$ is linear in $U$ or not.

Example 4.7 (The Trapezoidal Method.) This is a variant of the Crank-Nicolson Method, where middle point function evaluations $F\left(t^{n-\frac{1}{2}}, U^{n-\frac{1}{2}}\right)$ are replaced by averages $\frac{1}{2}\left[F\left(t^{n}, U^{n}\right)+F\left(t^{n-1}, U^{n-1}\right)\right]$. The standard form of the method is

$$
\bar{\partial} U^{n}=-\frac{1}{2}\left[F\left(t^{n}, U^{n}\right)+F\left(t^{n-1}, U^{n-1}\right)\right], \quad n=1, \ldots, N,
$$

with $U^{0}=u(0)$. This is a very popular second order method which can be seen as a two-point RK-C method, namely the lowest order Lobatto IIIA method. However, as a collocation method, $q=2$ and (1.2) is not satisfied, as expected since the order of the method is two. We can still cast the method within the framework of Section 2 provided we regard it as a piecewise linear approximation $(q=1)$, in agreement with the Crank-Nicolson method, instead of quadratic $(q=2)$; this is choice made in [19]. To this end, let $U \in \mathcal{V}_{1}$ be the continuous piecewise linear function that coincides with $U^{n}$ at the nodes. Then (4.17) can be written as

$$
U^{\prime}(t)=-\frac{1}{2}\left[F\left(t^{n}, U\left(t^{n}\right)\right)+F\left(t^{n-1}, U\left(t^{n-1}\right)\right)\right], \quad n=1, \ldots, N
$$


It is a simple matter now to verify, by using the properties of the trapezoidal quadrature rule, that (4.18) is equivalent to

$$
\int_{J_{n}}\left\langle U^{\prime}, v\right\rangle d t=-\int_{J_{n}}\left\langle\Pi_{0} F(t, U), v\right\rangle d t \quad \forall v \in \mathcal{H}_{0}\left(J_{n}\right),
$$

where $\Pi_{0}:=P_{0} I_{1}, I_{1}$ is the interpolation operator at $t^{n}, t^{n-1}$ by piecewise linear functions and $P_{0}$ is the $L^{2}$ projection onto $\mathcal{H}_{0}\left(J_{n}\right)$. This is of the form (2.2).

According to Section 2.1 the definition of $\widehat{U} \in \mathcal{H}_{1}$ should be based on appropriate projection operators $\widehat{\Pi}_{1}$ which satisfy the key property (2.9). The obvious choice $\Pi_{1}=I_{1}$ satisfies (2.9), the reconstruction $\widehat{U}$ defined by (4.10) coincides with $U$ at $t^{n-1}$ and $t^{n}$, Theorem 2.2 is applicable and thus the estimator $\widehat{U}-U$ is given by

$$
\widehat{U}^{\prime \prime}=-\frac{1}{k_{n}}\left[F\left(t^{n}, U^{n}\right)-F\left(t^{n-1}, U^{n-1}\right)\right] .
$$

This is similar to the two-point estimator (4.15). We also observe that the reconstruction $\widehat{U}$ coincides with the RK-C interpretation of Trapezoidal method. Indeed,

$$
\widehat{U}^{\prime}(t)=-I_{1} F(\cdot, U)(t)=-I_{1} F(\cdot, \widehat{U})(t)
$$

because $U-\widehat{U}$ vanishes at $t^{n-1}$ and $t^{n}$. We explore this connection further in [3].

An alternative reconstruction $\widehat{U}$ leading to a three-point estimator consists of taking $\widehat{\Pi}_{1}$ the linear interpolant joining the values $\widehat{\Pi}_{0} F(\cdot, U)(t)$ at the midpoints $t^{n-\frac{3}{2}}$ and $t^{n-\frac{1}{2}}$. This operator also satisfies (2.9), $\widehat{U}$ coincides with $U$ at $t^{n-1}$ and $t^{n}$, and $\widehat{U}^{\prime \prime}$ reads

$$
\begin{aligned}
\widehat{U}^{\prime \prime}=\frac{1}{k_{n}+k_{n-1}}\left[\left(F\left(t^{n}, U^{n}\right)\right.\right. & \left.+F\left(t^{n-1}, U^{n-1}\right)\right) \\
& \left.-\left(F\left(t^{n-1}, U^{n-1}\right)+F\left(t^{n-2}, U^{n-2}\right)\right)\right]
\end{aligned}
$$

whence

$$
\widehat{U}^{\prime \prime}=\frac{2}{k_{n}+k_{n-1}}\left(\frac{U^{n}-U^{n-1}}{k_{n}}-\frac{U^{n-1}-U^{n-2}}{k_{n-1}}\right) .
$$

This is similar to the three-point estimator (4.16), and is the time estimator proposed recently by Lozinski et al [19] for the heat equation. 


\subsection{A Posteriori Error Estimates}

Subtracting (4.12) from the differential equation in (1.1), we obtain

$$
\hat{e}^{\prime}(t)+A e(t)=B(t, u(t))-B(t, U(t))+R_{U}(t)
$$

with

$$
R_{U}(t)=B(t, U(t))-\widehat{I}_{q} B(t, U(t)) ;
$$

compare with (3.13) and (3.14). This leads to the following optimal result, the proof of which is similar to that of Theorem 3.1 and thus omitted.

Theorem 4.1 (Error Estimates for Nonlinear Equations) Let the assumptions of $\S 3$ about $A, B, f$ and $u^{0}$ be valid. In addition, if (2.7) holds, then so does the global upper bound of Theorem 3.1 for q-stage $R K-C$ methods. The local lower bound in Theorem 2.1 as well as the expressions in Theorem 2.2 for $\widehat{U}-U$ are also valid.

\section{Explicit Conditions for $U, \widehat{U} \in V$}

In this section we discuss whether $\widehat{U}$ satisfies the regularity condition (2.11) assumed throughout the paper. We provide conditions on the data and the reconstruction which guarantee its validity for the time discrete case, although, (2.11) is always satisfied by fully discrete schemes for evolution PDEs. To be precise, we examine sufficient conditions on $U^{0}$ and $F\left(0, U^{0}\right)$ for

$$
\begin{gathered}
U(t) \in V \quad \forall t \in[0, T], \\
\widehat{\Pi}_{q} F(\cdot, U)(t) \in V \quad \forall t \in[0, T],
\end{gathered}
$$

for the class of methods of Section 2 for which the reconstruction reads

$$
\widehat{U}^{\prime}(t)=U^{n-1}-\int_{t^{n-1}}^{t} \widehat{\Pi}_{q} F(\cdot, U)(s) d s
$$

for an interpolation operator satisfying (2.8). This implies $\widehat{U}(t) \in V$ and thereby gives (2.11). We first study (5.2), and next we establish sufficient conditions for (5.1) depending on the class and nodes $\left\{t^{n, i}\right\}_{i=0}^{q}$. To avoid confusion, note that $t^{n, 0}$ is not necessarily assumed to belong to the interval $\left[t^{n-1}, t^{n}\right]$, to which $\left\{t^{n, i}\right\}_{i=1}^{q}$ belong. Correspondingly, we set $U^{n, 0}=U\left(t^{n, 0}\right)$ where $U$ by its definition is a function defined on $[0, T]$ which is polynomial of degree $q$ in each interval $J_{n}$. 
Lemma 5.1 (Regularity) If (5.1) holds and

$$
F\left(t^{n, 0}, U^{n, 0}\right) \in V
$$

for all $n \geq 1$, then

$$
\widehat{\Pi}_{q} F(t, U(t)) \in V \quad \forall t \in[0, T] .
$$

Proof Let $t \in J_{n}$. From the assumption $U(t) \in V$ it immediately follows that $U(t)$ can be expressed in terms of the Lagrange polynomials with coefficients belonging to $V$. Thus, by differentiation, it follows that $U^{\prime}(t) \in V$, for all $t \in J_{n}$. Consequently,

$$
F\left(t^{n, i}, U^{n, i}\right)=-U^{\prime}\left(t^{n, i}\right) \in V, \quad i=1, \ldots, q .
$$

Since $F\left(t^{n, 0}, U^{n, 0}\right) \in V$ by hypothesis, with $t^{n, 0} \neq t^{n, i}$ for $i>0$, we conclude that the interpolant $\widehat{\Pi}_{q} F(t, U(t))$ belongs to $V$, for all $t \in J_{n}$, as asserted, because it is a linear combination of Lagrange polynomials with coefficients $\left\{F\left(t^{n, i}, U^{n, i}\right)\right\}_{i=0}^{q}$.

We now turn our attention to (5.1), (5.2) and split the analysis according to the method. It is an interesting open question whether the following conditions can be weakened, thereby extending the applicability of our results herein.

\subsection{Runge-Kutta Collocation Method (Revisited)}

We examine two cases in accordance with the location of node $\tau_{1}$.

Case 1: $\tau_{1}>0$. We prove (5.1) provided

$$
U^{0} \in V \text {. }
$$

We argue that $U^{n-1} \in V$ implies $U(t) \in V$ for all $t \in J_{n}$ by induction on $n$. For $n=0$ the statement is void. Let $n \geq 1$ and observe that $I_{q-1} U(t) \in D(A)$ for $t \in J_{n}$, in view of (4.7), whence $U^{n, i} \in D(A) \subset$ $V$ for $i=1, \ldots, q$. Obviously, $U(t)$ is a linear combination of the nodal values $\left\{U^{n, i}\right\}_{i=1}^{q}$ and $U^{n-1}$; since by induction $U^{n-1} \in V$, we deduce that $U(t) \in V$ for all $t \in J_{n}$.

To apply Lemma 5.1 it remains to choose $t^{n, 0}$ judiciously and to verify (5.3). We consider two cases, depending on whether $\tau_{q}<1$ or $\tau_{q}=1$.

If $\tau_{q}<1$, then we choose $t^{n, 0}$ to be a collocation point in a consecutive internal to $J_{n}$. If $n=1$ we take $t^{1,0}=t^{2,1}$ to be the first collocation point of the next interval. If $n>1$, instead, we select $t^{n, 0}=t^{n-1, q}$ to be the last collocation point of the previous interval. 
In both cases, the choice $t^{n, 0}$ is acceptable for a posteriori error estimation because $F\left(t^{n, 0}, U^{n, 0}\right) \in V$ is at our disposal. The RK GaussLegendre family is a key example (Example 4.1). An explicit formula of a reconstruction $\widehat{U}$ provided by the above procedure is given in the case of the Crank-Nicolson method with three-point estimator (Example 4.6).

If $\tau_{q}=1$, then $U^{n} \in D(A) \subset V$ whence, invoking (4.7) again, $F\left(t^{n}, U^{n}\right) \in V$ for all $n \geq 1$. Therefore, we can now choose $t^{n, 0}=t^{n-1}$ for $n \geq 2$ and obtain (5.2) for $t \geq t^{1}$. For the first interval we could take $t^{1,0}=0$ provided $F\left(0, U^{0}\right) \in V$ or the first collocation point of the second interval, $t^{1,0}=t^{2,1}$, otherwise. The RK Radau IIA is a key example for $q>1$ and $U^{0} \in V$ (Example 4.2).

Case 2: $\tau_{1}=0$. We prove (5.1) provided

$$
F\left(0, U^{0}\right) \in V
$$

and $F$ is continuous. Condition (5.5) is necessary for $\widehat{U}(t) \in V$ for $0 \leq t \leq t^{1}$ according to (4.10), but implies (5.1) only when $\tau_{q}=1$. In fact, in this case $U(t)$ is continuously differentiable because

$$
U^{\prime}\left(t^{n+}\right)=-F\left(t^{n+}, U\left(t^{n+}\right)\right)=-F\left(t^{n-}, U\left(t^{n-}\right)\right)=U^{\prime}\left(t^{n-}\right)
$$

due to the continuity of $U$ and $F$. We can argue by induction on $n \geq 0$ that $U^{\prime}\left(t^{n}\right) \in V$. For $n=0$ the statement is (5.5). For $n>0$ we see that $U^{n, i} \in V$ for $i=1, \ldots, q$ and $U^{\prime}\left(t^{n-1}\right) \in V$ by induction. This implies that $U(t) \in V$ for all $t \in J_{n}$ because it is a polynomial of degree $\leq q$, whence $U^{\prime}\left(t^{n}\right) \in V$. RK Lobatto IIIA methods are a key example for $q>2$ (Example 4.3).

On the other hand, if $\tau_{q}<1$, there is no smoothing property and neither $U(t)$ nor $\widehat{U}(t)$ are well defined for $t \geq t^{1}$. A relevant example is the explicit Euler method $U^{n}=U^{n-1}-k_{n} A U^{n-1}+k_{n} f\left(t^{n-1}\right)$, for which $U^{0} \in D(A)$ gives $U^{1} \in H$.

As a final remark note that in the case of Trapezoidal Method (Example 4.7) with $U$ considered as piecewise linear function, the method is not a pure RK-C method. Thus it is not covered by the previous cases. On the other hand it is a simple matter to check that $U$ and $\widehat{\Pi}_{q}$ do satisfy (5.1), (5.2), provided $F\left(0, U^{0}\right) \in V$.

\subsection{Continuous Galerkin Method (Revisited)}

We finally modify the $L^{2}$-projection operator $P_{q}$ of cG and so construct an interpolation operator $\widehat{\Pi}_{q}$ satisfying the key property $(2.8)$ as well as the requisite properties for (5.1) and (5.2). 
Let $v$ be a smooth function and $\tilde{v}=P_{q-1} v$. We define $\widehat{\Pi}_{q} v \in$ $\mathcal{V}_{q}\left(J_{n}\right)$ to be the interpolant of $\tilde{v}$ at the $q$ Gauss points of $J_{n}$ plus the last Gauss point of $J_{n-1}$ if $n>1$ or the first Gauss point of $J_{2}$ if $n=1$; hence the fundamental property (2.8) holds. In view of Remark 2.2 we need to show that the terms appearing on the right-hand side of (2.20) are of optimal order. This property is trivially verified when $\widehat{\Pi}_{q}$ is a simple interpolation operator as in the case of RK-C methods above. In our case, however, we will show in the sequel that

$$
v(t)-\widehat{\Pi}_{q} v(t)=O\left(k^{q+1}\right) \quad \forall t \in J_{n},
$$

with $k=\max \left(k_{n-1}, k_{n}, k_{n+1}\right)$.

Let $\tilde{I}_{q} v$ be the interpolant of $v$ at the $q$ Gauss points of $J_{n}$ plus the last Gauss point of $J_{n-1}$. Since $v-\tilde{I}_{q} v=O\left(k^{q+1}\right)$ on $J_{n}$, it remains to show that $\tilde{I}_{q} v-\widehat{\Pi}_{q} v=O\left(k^{q+1}\right)$ on $J_{n}$. This will follow upon showing the nodal superconvergence

$$
\tilde{v}\left(t^{n, i}\right)-v\left(t^{n, i}\right)=O\left(k^{q+1}\right),
$$

where $t^{n, i}, i=1, \ldots, q$, are the Gauss points of $J_{n}$. This result being local applies as well to the two consecutive intervals to $J_{n}$, and so the additional Gauss point employed in defining both $\widehat{\Pi}_{q}$ and $\tilde{I}_{q}$. Since (5.6) is valid at $q+1$ distinct points, the asserted estimate $\tilde{I}_{q} v-\widehat{\Pi}_{q} v=O\left(k^{q+1}\right)$ follows.

Let $\ell_{n, i}$ be the Lagrange polynomial of degree $q-1$ so that $\ell_{n, i}\left(t^{n, j}\right)=$ $\delta_{i j}$. Then

$$
\begin{aligned}
\int_{J_{n}} \ell_{n, i} v d t & =\int_{J_{n}} \ell_{n, i} P_{q-1} v d t=\sum_{j=1}^{q} w_{n, j}\left(\ell_{n, i} P_{q-1} v\right)\left(t^{n, j}\right) \\
& =w_{n, i} P_{q-1} v\left(t^{n, i}\right)
\end{aligned}
$$

whence

$$
P_{q-1} v\left(t^{n, i}\right)=\frac{1}{w_{n, i}} \int_{J_{n}} \ell_{n, i} v d t
$$

Note also that

$$
\int_{J_{n}} \ell_{n, i} d t=w_{n, i} \Rightarrow \frac{1}{w_{n, i}} \int_{J_{n}} \ell_{n, i} d t=1
$$

By Taylor expansion we have

$$
v(t)-v\left(t^{n, i}\right)=Q\left(t-t^{n, i}\right)+\frac{\left(t-t^{n, i}\right)^{q+1}}{(q+1) !} v^{(q+1)}(\xi(t)),
$$


where $Q\left(t-t^{n, i}\right)$ is a polynomial of degree at most $q$ which contains only factors of $\left(t-t^{n, i}\right)$. Combining the aforementioned ingredients, and using the fact that Gauss quadrature integrates polynomials of degree $2 q-1$ exactly, we obtain

$$
\begin{gathered}
\tilde{v}\left(t^{n, i}\right)-v\left(t^{n, i}\right)=P_{q-1} v\left(t^{n, i}\right)-v\left(t^{n, i}\right)=\frac{1}{w_{n, i}} \int_{J_{n}} \ell_{n, i}\left(v(t)-v\left(t^{n, i}\right)\right) d t \\
=\frac{1}{w_{n, i}} \int_{J_{n}} \ell_{n, i}\left(Q\left(t-t^{n, i}\right)+\frac{\left(t-t^{n, i}\right)^{q+1}}{(q+1) !} v^{(q+1)}(\xi(t))\right) d t
\end{gathered}
$$

Since $Q\left(t-t^{n, i}\right)$ vanishes at $t^{n, i}$, we deduce

$$
\int_{J_{n}} \ell_{n, i}\left(Q\left(t-t^{n, i}\right) d t=\sum_{j=1}^{q} w_{n, j} \ell_{n, i}\left(t^{n, j}\right) Q\left(t-t^{n, i}\right)\left(t^{n, j}\right)=0\right.
$$

whence (5.6) follows easily

$$
\tilde{v}\left(t^{n, i}\right)-v\left(t^{n, i}\right)=\frac{1}{w_{n, i}} \int_{J_{n}} \ell_{n, i} \frac{\left(t-t^{n, i}\right)^{q+1}}{(q+1) !} v^{(q+1)}(\xi(t)) d t=O\left(k^{q+1}\right) .
$$

We finally recall that the interpolation operator $\widehat{\Pi}_{q}$ verifies $(2.8)$, and observe that $U$ satisfies (5.1) provided $U^{0} \in V$. To prove this, note that $P_{q-1} A U(t)=A U(t)$ at $q$ distinct points in $J_{n}$ because $P_{q-1}$ is the $L^{2}$-projection onto polynomials of degree at most $q-1$. Even though these points are not a priori known, we can argue as in Case 1 $\left(0<\tau_{1}<\tau_{q}<1\right)$ above to infer that $U(t), \widehat{U}(t) \in V$ for all $t \in[0, T]$.

\section{References}

1. Akrivis, G., Makridakis, Ch.: Galerkin time-stepping methods for nonlinear parabolic equations. M ${ }^{2}$ AN Math. Model. Numer. Anal. 38, 261-289 (2004)

2. Akrivis, G., Makridakis, Ch., Nochetto, R.H.: A posteriori error estimates for the Crank-Nicolson method for parabolic equations. Math. Comp. 75, 511-531 (2006)

3. Akrivis, G., Makridakis, Ch., Nochetto, R.H.: Galerkin and Runge-Kutta methods: unified formulation, a posteriori estimates and superconvergence. To appear.

4. Aziz, A.K., Monk, P.: Continuous finite elements in space and time for the heat equation. Math. Comp. 52, 255-274 (1989)

5. Bangerth, W., Rannacher, R.: Adaptive Finite Element Methods for Differential Equations. Lectures in Mathematics ETH Zürich, Birkhäuser Verlag, Basel, 2003.

6. Crouzeix, M.: Sur l'approximation des équations différentielles opérationelles linéaires par des méthodes de Runge-Kutta. Thèse, Université de Paris VI, 1975 . 
7. Crouzeix, M., Mignot, A.L.: Analyse Numérique des Équations Différentielles. $2^{\text {nd }}$ ed., Masson, Paris, 1989.

8. Davis, P.J., Rabinowitz, P.: Methods of Numerical Integration. $2^{\text {nd }}$ ed., Academic Press, New York, 1984.

9. Eriksson, K., Johnson, C.: Adaptive finite element methods for parabolic problems. I. A linear model problem. SIAM J. Numer. Anal. 28, 43-77 (1991)

10. Eriksson, K., Johnson, C., Larsson, S.: Adaptive finite element methods for parabolic problems. VI. Analytic semigroups. SIAM J. Numer. Anal. 35, 1315-1325 (1998)

11. Eriksson, K., Johnson, C., Logg, A.: Adaptive computational methods for parabolic problems. Encyclopedia of Computational Mechanics, Edited by E. Stein, R. de Borst and T. J. R. Hughes, 1-44, J. Wiley \& Sons, Ltd., New York, 2004.

12. Estep, D., French, D.: Global error control for the Continuous Galerkin finite element method for ordinary differential equations. RAIRO Modél. Math. Anal. Numér. 28, 815-852 (1994)

13. Estep, D., Larson, M., Williams, R.: Estimating the error of numerical solutions of systems of reaction-diffusion equations. Mem. Amer. Math. Soc., 146, no. 696, 109 pp., (2000)

14. Giles, M.B., Süli, E.: Adjoint methods for PDEs: a posteriori error analysis and postprocessing by duality. Acta Numer. 11, 145-236 (2002)

15. Hairer, E., Nørsett, S.P., Wanner, G.: Solving Ordinary Differential Equations I: Nonstiff Problems. Springer Series in Computational Mathematics v. 8, Springer-Verlag, Berlin, $2^{\text {nd }}$ revised ed., 1993, corr. $2^{\text {nd }}$ printing, 2000.

16. Hairer, E., Wanner, G.: Solving Ordinary Differential Equations II: Stiff and Differential-Algebraic Problems. Springer Series in Computational Mathematics v. 14, Springer-Verlag, Berlin, $2^{\text {nd }}$ revised ed., 2002.

17. Houston, P., Süli, E.: $h p$-adaptive discontinuous Galerkin finite element methods for first-order hyperbolic problems. SIAM J. Sci. Comput. 23, 1226-1252 (2001)

18. Lakkis, O., Nochetto, R.H.: A posteriori error analysis for the mean curvature flow of graphs. SIAM J. Numer. Anal. 42, 1875-1898 (2005)

19. Lozinski, A., Picasso, M., Prachittham, V.: An anisotropic error estimator for the Crank-Nicolson method: Application to a parabolic problem. SIAM J. Sci. Comput. (to appear).

20. Lubich, Ch., Ostermann, A.: Runge-Kutta methods for parabolic equations and convolution quadrature. Math. Comp. 60, 105-131 (1993)

21. Lubich, Ch., Ostermann, A.: Runge-Kutta approximation of quasi-linear parabolic equations. Math. Comp. 64, 601-627 (1995)

22. Makridakis, Ch., Nochetto, R.H.: Elliptic reconstruction and a posteriori error estimates for parabolic problems. SIAM J. Numer. Anal. 41, 1585-1594 (2003)

23. Makridakis, Ch., Nochetto, R.H.: A posteriori error analysis for higher order dissipative methods for evolution problems. Numer. Math. 104, 489-514 (2006)

24. Nochetto, R.H., Savaré, G., Verdi, C.: A posteriori error estimates for variable time-step discretizations of nonlinear evolution equations. Comm. Pure Appl. Math. 53, 525-589 (2000)

25. Shampine, L.F.: Error estimation and control for ODEs. J. Sci. Comput. 25, $3-16(2005)$ 
26. de Swart, J.J.B., Söderlind, G.: On the construction of error estimators for implicit Runge-Kutta methods. J. Comput. Appl. Math. 86, 347-358 (1997)

27. Thomée, V.: Galerkin Finite Element Methods for Parabolic Problems. $2^{\text {nd }}$ ed., Springer-Verlag, Berlin, 2006.

28. Zadunaisky, P.E.: On the estimation of errors propagated in the numerical integration of ordinary differential equations. Numer. Math. 27, 21-39 (1976) 